\title{
Determinación del sustrato para la germinación de semillas de lúcuma (Lucuma obovata bkb) patrón con fines de injertación en Cañasbamba, Yungay, Áncash
}

Determination of substrate for seed germination of lucuma (lucuma obovata hkb) pattern with the purpose grafting in Cañasbamba, Yungay, Ancash

\author{
Sacramento Díaz León ${ }^{1}$, Nuri Maguiña Ropón ${ }^{1}$, Nelly Caycho Medrano ${ }^{1}$, \\ Walter VÁsquez CruZ ${ }^{1}$ y Francisco Espinoza Montesinos ${ }^{1}$
}

\section{RESUMEN}

Los objetivos fueron: a) determinar el sustrato adecuado para la germinación de la radícula de semillas de lúcumo (patrón) con fines de injertación con yemas de lúcuma (Lucuma obovata $\mathrm{HKB}$ ) variedad seda, el porcentaje de las radículas de calidad de la lúcuma patrón en los diferentes sustratos; el porcentaje de prendimiento de las radículas de lúcuma injertadas con la variedad Seda; b) encontrar el periodo de tiempo hasta obtener un plantón (planta injertada) para ser trasplantado a campo definitivo. Los sustratos evaluados fueron: Compost (T1), Arena (T2), Arena + Compost (proporción de 3:1 respectivamente), Arena + Compost (proporción de 1:1 respectivamente). La técnica utilizada fue la raíz invertida y se realizó el injerto tipo púa con las yemas de lúcuma variedad seda. El tratamiento T3 (3:1) resultó ser el sustrato más adecuado para la germinación de la radícula de lúcumo (Lucuma obovata HKB), con fines de injertación; se obtuvo que el 97\% del tratamiento T3, fue radículas de calidad para realizar el injerto, a partir de la germinación de semillas de la lúcuma patrón. Se determinó que a los 8 meses, se obtiene un plantón (planta injertada) apto para ser trasplantado en el campo definitivo.

Palabras clave: sustrato; semilla; yema; injerto.

\begin{abstract}
The objetives to determine the substrate suitable for the germination of the radicle of seeds of lucuma (pattern) purposes of grafting buds of lucuma (Lucuma obovata HKB) silk variety, the percentage of the rootlets of the pattern in the different substrates lucuma quality; the percentage of arrest of the rootlets of lucumo grafted with the silk variety; and find the time period until a sit-in (grafted plant) to be transplanted to final field. The substrates evaluated were: Compost (T1), sand (T2), Sandy Compost (3:1 ratio respectively), sand Compost (1:1 ratio respectively). The technique used was inverted root and performed the graft type pua lucuma fingertips variety silk. T3 (3:1) treatment turned out to be the most suitable substrate for germination of the radicle of lucuma (Lucuma obovata HKB), for grafting purposes, was that 97\% of T3 treatment, quality radicles were to perform the graft from the germination of seeds of
\end{abstract}

1 Universidad Nacional «Santiago Antúnez de Mayolo». Huaraz, Perú. 
the eggfruit pattern, It was determined that at 8 months, gets a sit-in (grafted plant) suitable to be transplanted in the final field.

Keywords: substrate; seed; bud; bud grafting.

\section{ICHIKLLACHAW}

Kay uryachaw willakun lukma murukunapa radikula alli hiqayninpaq, tsay lukmapa shunqunkunawan inhirtasiyunninraykur (Lukma obovata HKB) siida kaq laaya lukma radikulanta alli kananpaq, rikaarishun llapan huklaaya sustratukunachaw: llapan pursintahinchaw kay radikulakunaqa inhirtadu siida kaq laayawan tiyimpupa piryudunta tarinapaq, planta hawayninta awqanapaq (inhirtada) kikin chakraman hukwarishqa patsakaananpaq, sustratukuna chanintsaynin karqan: Compost (T1), aqu (T2), aqu + Compost (3:1pa prupursiyunnin), aqu + compost (1:1 pa prupursiyunnin). Tiknikanpis watsu tikrakashqawanmi rurakashqa, hinaman puwa laaya inhirtutam rurakashqa lukmapa shunqunkunawan siida kaq laayakunawan.

Tsay tratamiyintuqa kanaq T3 (3:1) kaynaw yarqunaq atska shumaq kaq sustrakukuna lukmapa radikulan hiqamunanpaq (Lukma obovata HKB) inhirtasiyun kananpaq, tsay yarqunaq llapan tratamiyintupita T3, llapan alli kaq radikulakuna kanaq inhirtukuna alli ruranapaq, puntata shumaq murukunapa hiqaynin lukmapita qallaykur. Llapan plantakuna inhirtuwan alli kananpaq 8 killapaq churakarqan, tsaypiqmi huk alli plantun yarqun (inhirtada planta) huk kikin chakrallamanna shumaq yapay plantanapaq.

Pushaq shimikuna: sustratu; muru; murupa shunqun; inhirtu.

\section{INTRODUCCIÓN}

El lúcumo es nativo de los valles interandinos del Perú y Ecuador (SIICEX, 2008). La lúcuma ha sido utilizada en la alimentación humana desde épocas anteriores al incanato, como lo evidencian los huacos de las culturas: Mochica, Chimú, Nazca y Lambayeque. En nuestro país, los departamentos de Áncash, Cajamarca, Ayacucho y Lima, constituyen el principal centro de origen del Lúcumo (Avalos, 2008). En la sierra todavía este cultivo continúa sin mayores cambios; en la costa actualmente ya se utiliza adecuados biotipos y manejo.

Según la mitología andina, su nombre proviene de una mujer altiva, orgullosa, de gran belleza y de poca paciencia. Lúcuma, es sinónimo de una de las frutas más preciadas de la gastronomía peruana y con creciente presencia en las diversas cocinas del mundo. Su notoriedad aumenta desmedidamente gracias a los recientes descubrimientos de sus enormes propiedades medicinales y nutritivas (Avalos, 2008).

Debido a su potencial en el campo de la gastronomía y su valor nutritivo y medicinal, actualmente la lúcuma se exporta a diversos países miembros de la Unión Europea y también hacia los Estados Unidos, donde se ha intentado sin éxito alguno implantar su producción. En estas latitudes, esta especie llega en sus diversas presentaciones: pulpa, harina de lúcuma y lúcuma fresca. Todo un abanico de posibilidades para generar divisas. 
La diversidad genética es muy apreciable. Existen dos tipos: «lúcuma seda» y «lúcuma palo» que corresponde a la menor o mayor dureza del mesocarpio o pulpa de la fruta madura. Se denomina Lúcuma de Seda a los frutos que al madurar presentan textura suave; en caso contrario, los frutos son llamados Lúcuma Palo. También existe diversidad por tamaño y forma de la fruta, color de la cáscara (de verde a verde amarillento) y de la pulpa (de amarillo claro a amarillo intenso) y aroma de la fruta (Universidad del Pacífico, 2000).

La lúcuma se puede propagar por semilla (conocida también como propagación botánica) y/o por propagación vegetativa. Franciosi (1992) afirma que la mayoría de las plantas de lúcumo que hay en el Perú proceden de semilla y, por lo tanto, muestran una extrema variabilidad tanto en las características de las plantas (tamaño, vigor, etc.) como en las de los frutos en cuanto a tamaño, forma, calidad y color de la pulpa, número y tamaño de semillas, consistencia de la pulpa que le permiten una mayor tolerancia al transporte.

La lúcuma, uno de nuestros frutales nativos, no cuenta con estudios referidos a su propagación; se encontró información de Chile, una de las potencias en frutales, la Estación Experimental «La Palma» de la Escuela de Agronomía de la Universidad Católica de Valparaíso realizo un novedoso injerto en la raíz, desconocido aún en nuestro país.

La mayoría de plantas de lúcuma establecidas en el Callejón de Huaylas son francas, solo se propagaron por semillas y se establecieron en campo definitivo; actualmente debido al incremento de la demanda se realiza mejoramientos genéticos mediante la técnica del injerto.

Los tipos de injerto a emplearse son el terminal simple o el terminal de doble lengüeta. La altura del injerto es de $20 \mathrm{~cm}$. por encima del cuello de la planta, dejando unas $3 \mathrm{a}$ 4 hojas que ayudarán a mejorar el prendimiento de la yema. Cuando la injertación ha sido bien realizada y en la época apropiada, las primeras yemas prendidas comienzan a desarrollarse a los 30 días y el prendimiento de las siguientes puede durar hasta 60 días o más. Las plantas están listas para ser llevadas al campo definitivo cuando las hojas formadas, por lo menos seis, estén completamente desarrolladas y maduras; lo cual puede lograrse seis meses después del injerto (Franciosi, 1992).

La propagación del lúcumo se hace por injerto sobre portainjertos, aunque con algunas dificultades relacionadas al lento crecimiento de las plántulas, las cuales necesitan 18 meses para tener un plantón (planta injertada lista para ser llevada a campo definitivo) con un lento retorno del capital invertido, pérdida de tiempo y la obtención de plantones de mala calidad, por permanecer demasiado tiempo en la bolsa plástica. Para la lúcuma, uno de los frutales nativos que no cuenta con estudios referidos a las técnicas de su propagación en nuestro país, se encontró información en la Estación Experimental «La Palma», Escuela de Agronomía de la Universidad Católica de Valparaíso de Chile, un novedoso injerto en la raíz de semillas, así que se decidió realizar este trabajo de investigación, tomando en cuenta para ello el sustrato germinativo, que va de la mano con la semilla y la yema (Gardizábal y Valenzuela, 1984). 
Gardizábal y Valenzuela (1984) refieren que utilizó el sustrato 4:1 de arena y turba respectivamente, obteniendo hasta un $80 \%$ de prendimiento, pero dando las mejores condiciones ya que utilizaron una estructura protegida con mesones de propagación y un sistema de neblina artificial.

La semilla de lúcuma con las condiciones de invernadero que se le dieron, germinó a un mes, y a los 15 días se realizó el injerto en la radícula. A los dos meses se observó los primeros brotes de $1 \mathrm{~cm}$. de longitud, y a los tres meses se vio brotes bien desarrollados de $3 \mathrm{~cm}$. de longitud. A los tres meses y medio se tenía brotes de 7,5 cm. de longitud. A los 4 meses el brote creció a $15 \mathrm{~cm}$. Se podó los brotes laterales de algunas yemas y a los seis meses el plantón tenía $40 \mathrm{~cm}$. de longitud. A los ocho meses el plantón estuvo listo para campo definitivo con $60 \mathrm{~cm}$. de longitud y 1,8 cm. de diámetro.

Diversas experiencias de viveristas mencionan que con la técnica convencional tomando como base desde la germinación de las semillas, hasta obtener el grosor adecuado para el injerto se requiere 8 meses, posteriormente son 7 meses adicionales para la obtención de un plantón listo para campo definitivo. En el Perú no se cuenta con esta tecnología.

Los objetivos del trabajo de investigación fueron: a) Determinar el sustrato adecuado para la germinación de la radícula de lúcumo (Lúcuma obovata HKB), con fines de injertación con yemas de la variedad seda, el porcentaje de las radículas de calidad de la lúcuma patrón en los diferentes sustratos, el porcentaje de prendimiento de las radículas de lúcuma patrón injertadas con la variedad seda y b) Encontrar el periodo de tiempo hasta obtener un plantón (planta injertada) apto para ser llevado a campo definitivo.

\section{MATERIALES Y MÉTODOS}

El estudio se realizó en el CIPA - Cañasbamba, a una altitud de $2284 \mathrm{msnm}$, latitud

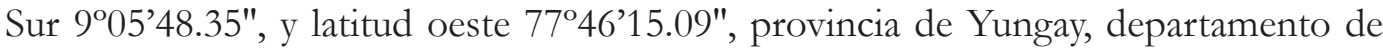
Áncash, desde noviembre del 2012 hasta julio del 2014.

El material vegetal: Semillas y yemas de lúcumo. Insumos: Ácido giberélico (Projibb), regulador de crecimiento (Root Hor), desinfectante (Benlate), cicatrizante (Sanix), alcohol, arena y compost.

Se utilizó el Diseño Completamente al Azar (DCA), con 4 tratamientos y 3 repeticiones, el tamaño de la unidad experimental, que constó de 10 semillas de lúcuma patrón, haciendo un total de 120 semillas que se empleó en el trabajo de investigación.

Tabla 1. Composición y proporcionalidad del sustrato

\begin{tabular}{ccc}
\hline TRATAMIENTO & COMPOSICION DEL SUSTRATO & $\%$ DE \\
\hline T1 & Compost (testigo) & $100 \%$ compost \\
T2 & Arena & $100 \%$ arena \\
T3 & Arena + compost $(3: 1)$ & $75 \%$ arena $+25 \%$ compost \\
T4 & Arena + compost $(1: 1)$ & $50 \%$ arena $+50 \%$ compost \\
\hline
\end{tabular}

Se construyó un invernadero de $2 \mathrm{~m}$. de ancho, 2,5 m. de largo y 2,20 m. de altura y se 
forró la parte externa con plástico transparente, lo cual dio las condiciones necesarias para la germinación (mayor temperatura y humedad). En el interior se construyó una cama de germinación de 0,90 m. de ancho, 2,00 m. de largo y 0,40 m. de profundidad. Se realizó las comparticiones de la cama de germinación con el triplay teniendo en cuenta los distanciamientos del tratamiento. El sustrato se desinfectó con agua hirviendo, vertiéndola sobre el sustrato previamente humedecido con agua limpia, con la finalidad de eliminar agentes patógenos; luego se dejó enfriar.

Se seleccionaron las semillas, las más grandes, libres de plagas, enfermedades y que no presenten daños mecánicos. Se retiró la cubierta de la semilla (cáscara). Se desinfectó las semillas remojándolas por 1 hora en Benlate al 2 por mil).

Las semillas previamente a la siembra se remojaron por 5 minutos en una solución de Ácido Giberélico (Progib), disolviéndose 1 gr. del producto en $10 \mathrm{~cm}^{3}$ de alcohol y, se vertió en un recipiente con 10 litros de agua y luego se procedió a la siembra en la cama germinadora alineándolo en filas y columnas. Los riegos se realizaron periódicamente cada vez que fue necesario teniendo en cuenta las condiciones climáticas del invernadero.

El injerto se realizó a los 45 días después de la siembra, con yemas de la variedad Seda (yema de huevo). Se sacó las semillas del sustrato y se las enjuagó en agua limpia, sin dañar las raicillas, procediéndose a realizar el corte transversal de la raíz a $1 \mathrm{~cm}$. desde la base de la semilla, luego se efectuó un corte longitudinal a través de los pecíolos cotiledonarios en sentido contrario a su división para evitar así la apertura de la semilla en dos mitades. Se seleccionaron plumas en que el diámetro coincidiera con el diámetro de la radícula. Se procedió con el injerto tipo púa (injerto de raíz invertida). Se insertó la pluma en la radícula tratando de hacer coincidir el cambium de la yema con el cambium de la raíz de la semilla. Luego se amarró con una liga delgada, dejando espacios libres para que puedan emerger y crecer las raíces. Los injertos fueron plantados en bolsas de polietileno. Se aplicó un regulador de crecimiento (enraizador) a un mes después del injerto: Root Hor (18 ml. en 10 lts. de agua) para promover el crecimiento de las raíces. Se verificó que los injertos queden visibles, para evitar la deshidratación de las yemas. La humedad del sustrato se tuvo a capacidad de campo.

\section{RESULTADOS}

1. Diámetro de la radícula

Tabla 2. Prueba de comparación de medias de Duncan para el diámetro $(\mathrm{cm}$.) de la radícula del efecto de las proporciones del sustrato

\begin{tabular}{clc}
\hline Orden de mérito & \multicolumn{1}{c}{ Tratamiento } & Promedio (cm.) \\
\hline I & T3 = Arena + Compost (3:1) & 0.48 \\
II & T2 = Arena & $0.36 \mathrm{~A}$ \\
III & T4 $=$ Arena + Compost $(1: 1)$ & $0.36 \mathrm{~A}$ \\
IV & T1 $=$ Compost & 2.01 \\
\hline
\end{tabular}


No hay diferencias estadísticas significativas entre los promedios con la misma letra. El T3 resultó el sustrato más adecuado para la germinación de las semillas y las radículas obtenidas fueron de calidad para realizar el injerto, mostrando diferencias significativas con los demás tratamientos. El T2 y el T4 no mostraron diferencias estadísticas significativas con respecto al diámetro de radícula.

\section{Longitud de radícula}

Tabla 3. Prueba de comparación de medias de Duncan para la longitud de radícula (cm.) del efecto de las proporciones del sustrato

\begin{tabular}{clc}
\hline Orden de mérito & \multicolumn{1}{c}{ Tratamiento } & Promedio $(\mathrm{cm})$. \\
\hline I & T2 $=$ Arena & 12.18 \\
II & T4 = Arena + Compost $(1: 1)$ & $10.40 \mathrm{~A}$ \\
III & T3 $=$ Arena + Compost $(3: 1)$ & $10.39 \mathrm{~A}$ \\
IV & T1 $=$ Compost & 2.13 \\
\hline
\end{tabular}

No hay diferencias estadísticas significativas entre los promedios con la misma letra. Con el T2 se obtuvo mayor longitud de radícula y mostró diferencias estadísticas significativas, con los demás tratamientos.

\section{Porcentaje de prendimiento}

El porcentaje de prendimiento se evaluó al tercer mes después de la injertación. De 68 plantas injertadas prendieron 41 yemas, constituyendo el 60\% de prendimiento. Se podría haber obtenido mayor porcentaje de germinación si se le hubiese dado mayores condiciones de infraestructura, un tipo de neblina artificial (cada 10 minutos, 1 minuto de neblina).

\section{Tiempo de prendimiento}

Las semillas de lúcuma germinaron al mes de la siembra bajo condiciones de invernadero y 15 días después se realizó el injerto en la radícula. A los dos meses se observó los primeros brotes de $1 \mathrm{~cm}$. de longitud, a los tres meses se vio brotes bien desarrollados de $3 \mathrm{~cm}$. de longitud, a los tres meses y medio se tenía brotes de 7,5 cm. de longitud y a los 4 meses el brote creció a $15 \mathrm{~cm}$. Se podó los brotes laterales de algunas yemas y a los seis meses el plantón tenía $40 \mathrm{~cm}$. de longitud. A los ocho meses el plantón estuvo listo para campo definitivo con $60 \mathrm{~cm}$. de longitud y 1,8 cm. de diámetro.

\section{DISCUSIÓN}

Con el tratamiento T3 = Arena + compost (3:1), se tuvo grosor de radícula apropiado para realizar la injertación. Con respecto al porcentaje de prendimiento se obtuvo el $60 \%$, bajo las condiciones de invernadero en que se realizó el experimento. Según Gardizábal (1993) se utilizó como sustrato de enraizamiento una mezcla de arena gruesa y turba en una relación $4: 1$, obteniendo hasta un $80 \%$ de prendimiento, pero dando 
mejores condiciones ya que utilizaron una infraestructura protegida, con mesones de propagación y un sistema de neblina artificial. En ambos casos hay coincidencia en que los mejores resultados se obtienen con sustratos de mayor proporción de arena.

Este novedoso injerto de raíz invertida acorta el tiempo de obtención de un plantón a 8 meses, con respecto a la técnica convencional, indicada por diferentes viveristas. Tomando como base desde la germinación de las semillas hasta obtener el grosor adecuado para el injerto se requiere 8 meses; posteriormente son 7 meses adicionales para la obtención de un plantón listo para campo definitivo. Franciosi (1992) indica que las semillas de lúcuma germinan a los 20 - 25 días, estando las plantas listas para injertar cuando tienen una altura promedio de $25 \mathrm{~cm}$. y un diámetro de $1 \mathrm{~cm}$. aproximadamente. Así mismo transcurren desde la siembra a la injertación de 9 a 10 meses y falta adicionar el tiempo de prendimiento hasta que esté listo el plantón para campo definitivo.

\section{CONCLUSIONES}

El T3 (3 arena: 1compost) es el sustrato más adecuado para la germinación de la radícula de Lúcumo (Lúcuma obovata HKB), con fines de injertación.

Con el T3 (3 arena: 1 compost) se obtuvo el 97\% de radículas de calidad para realizar el injerto, seguido del tratamiento T4 (1 arena: 1 compost) que llegó a 73\%, el tratamiento T2 (arena) con 57\% y el T1 (compost) con 3\%. Este mismo orden presentó la variable número de semillas germinadas.

Se observó un $60 \%$ de prendimiento de las radículas de lúcuma injertada con la variedad seda.

Con la técnica de injerto en raíz invertida se obtuvo un plantón para campo definitivo a los 8 meses después del injerto acortándose el tiempo con respecto al injerto convencional.

\section{REFERENCIAS BIBLIOGRÁFICAS}

Ávalos, Carlos. 2008. La lúcuma, fruta que cura. Perú. 5. <http:/ /www.generaccion.com> [Consulta: 04-10-2012].

Franciosi, Rafael. 1992. El Cultivo de Lúcumo en el Perú. Lima: Editorial Fundeagro.

Gardizábal, Francisco y Valenzuela, Luis. 1984. Nuevas técnicas de propagación en lúcumo. Valparaíso: Universidad de Valparaiso.

MINAG. 2010. Cultivo de lúcumo. Lima. < http://www.minag.gob.pe> [Consulta: 04-102012].

Universidad del Pacífico. 2000. Seminario de Agro Negocios: Lúcuma. Lima. 55. <http:// www.up.edu.pe> [Consulta: 12-08-2012].

Sistema Integrado de Información de Comercio Exterior. 2008. Lúcuma. Lima. $<$ http://www.SIICEX.gob.pe> [Consulta: 12-08-2012]. 
|| Sacramento Díaz, Nuri Maguiña, Nelly Caycho, Walter Vásquez, Francisco Espinoza

Fecha de recepción: 15 de enero de 2015

Fecha de aceptación: 20 de marzo de 2015

\section{Correspondencia}

Sacramento Diaz León

neptali_45@hotmail.com 